\title{
Normobaric Oxygen Therapy in Acute Stroke: A Systematic Review and Meta-Analysis
}

\author{
Ammad Mahmood Sam Neilson Viveka Biswas Keith Muir \\ Institute of Neuroscience and Psychology, University of Glasgow, Glasgow, UK
}

\section{Keywords}

Acute stroke · Oxygen therapy · Neuroprotection in stroke ·

Systematic reviews

\begin{abstract}
Purpose: Normobaric oxygen (NBO) is potentially a readily accessible neuroprotective therapy. We undertook a systematic review to assess NBO in acute stroke. Methods: MEDLINE, EMBASE, and CENTRAL databases were searched to December 2020. Randomized controlled trials of NBO administered $<7$ days after stroke to normoxic patients with no other indication for oxygen were identified. Data on early neurological recovery; functional outcome; mortality; oxygen saturation, and imaging markers were collected. Findings: Fifteen publications involving 12 cohorts and 9,255 participants were identified. One study with 8,003 participants had low risk of bias, but the designs of smaller trials had limitations. Ninety-seven per cent of participants were in studies of low-flow oxygen ( $\leq 4 \mathrm{~L} / \mathrm{min}$ ). $82.8 \%$ had ischaemic stroke. Median time to treatment was $19.3 \mathrm{~h}$. Meta-analysis demonstrated no significant effect on: reduction in $\mathrm{Na}$ tional Institutes of Health Stroke Scale at 7 days in all stroke or ischaemic stroke only (mean difference $-0.16[-1.11$ to 0.80 ] and -0.73 [-3.54 to 2.08], respectively); modified Rankin scale at 3-6 months of follow-up (combined stan-
\end{abstract}

karger@karger.com www.karger.com/ced

Karger $\stackrel{\text { ' }}{5}$

GOPEN ACCESS
(C) 2022 The Author(s)

Published by S. Karger AG, Basel

This is an Open Access article licensed under the Creative Commons Attribution-NonCommercial-4.0 International License (CC BY-NC) (http://www.karger.com/Services/OpenAccessLicense), applicable to the online version of the article only. Usage and distribution for commercial purposes requires written permission. dardized mean difference [SMD] -0.08 [ -0.38 to 0.22$] ; 3$ months SMD -0.01 [ -0.03 to 0.029]; 6-month SMD -0.20 [-1.49 to 1.09$]$ ), or mortality (odds ratio 1.15 [0.87-1.53]). Discussion: The majority of patients were administered lowflow oxygen in the sub-acute phase. Intervention strategies targeted at modification of early tissue survival (higher oxygen delivery and administration at early time points when significant volumes of viable tissue persist) have not been tested adequately. Conclusion: Studies of NBO have shown no significant effect on early neurological recovery, functional outcome, or mortality in acute stroke. Oxygen has been predominantly low-flow and commenced in the subacute phase.

(c) 2022 The Author(s)

Published by S. Karger AG, Basel

\section{Introduction}

Low tissue oxygen delivery resulting from vessel occlusion is central to the bioenergetic failure that characterizes acute ischaemic stroke. Reflecting limited clinical data, current guidelines from the European Stroke Organization [1] and American Heart Association [2] do not advocate the routine use of supplemental oxygen for nonhypoxic patients (those in whom oxygen saturation is $\geq 95 \%$ ). Reperfusion with thrombolytic drugs or endovas- 
cular mechanical thrombectomy is highly effective but benefit is time-dependent and times to effect reperfusion may be several hours after treatment initiation. A treatment that is able to retard or prevent hypoxic cell death may therefore have potential value as a neuroprotectant in this population, as well as those for whom reperfusion is not indicated or is ineffective. Supplemental normobaric oxygen (NBO) is usually a readily available resource in acute hospital environments. In animal models, low levels of tissue oxygen pressure in the ischaemic penumbra are significantly improved by NBO therapy despite only mild increases in arterial oxygen content [3] indicating therapeutic potential even in the absence of restoring blood flow. In rodent models of permanent focal ischaemia, $100 \%$ oxygen commenced $5 \mathrm{~min}$ after induction of transient ischaemia (up to $3 \mathrm{~h}$ duration) resulted in smaller final infarct volumes than control [4], possibly due to persistence of salvageable penumbra. NBO-treated rats have shown attenuation of DWI lesions and smaller infarct size, and the combination of NBO and intravenous thrombolysis has been shown to be safe and effective in a rat model of stroke with near complete reperfusion, small infarct volumes and no difference in rates of haemorrhage and brain swelling $[5,6]$. This review aimed to identify all randomized controlled trials of NBO in patients diagnosed with acute stroke and examined the effect on early recovery, functional outcome, and mortality.

\section{Methods}

The protocol for the review has been registered with PROSPERO (CRD42018116250). MEDLINE, EMBASE, and CENTRAL databases were searched using MeSH terms and Boolean operators from inception to December 2020, search criteria are available in online supplementary materials (for all online suppl. material, see www.karger.com/doi/10.1159/000521027). Studies were identified by 2 researchers independently (A.M. and S.N.) by screening titles and abstracts, a third researcher (V.B.) resolved any discrepancies. The following inclusion and exclusion criteria were used:

- Population - patients with stroke in the last 7 days entered into randomized controlled trials of NBO. Quasi-randomized trials (e.g., allocation by date of birth) were accepted but clinical trials without randomization were excluded.

- Intervention - NBO administered $<7$ days after stroke (ischaemic or haemorrhagic) to normoxic patients with no other indication for oxygen therapy. This included NBO at any concentration or flow rate and for any length of time. Oxygen treatment at $2-4 \mathrm{~L} / \mathrm{min}$ delivered by nasal cannula was considered low flow; higher rates by mask were considered high flow.

- Comparison - control groups receiving either no oxygen or oxygen only given if clinically indicated

- Outcome - data were collected from published abstracts, articles, or clinical trials databases on study design, details of oxy- gen therapy, time to treatment from stroke onset, early recovery within 7 days; functional outcome within 9 months; mortality; oxygen saturation and imaging markers. Data from multiple publications from the same cohort were combined rather than being treated separately. Efforts were made to contact authors where initially retrieved data were insufficient.

For the purposes of meta-analysis, mean difference or standardized mean difference was calculated for functional outcome, early neurological recovery, and oxygen saturation; and odds ratio was calculated for mortality. A subgroup analysis for ischaemic stroke only was carried out for these outcomes where data allowed. The following statistical transformations were employed. Mean and variance were calculated from median, range, and IQR as per the methodology of Hozo et al. [7] and Luo et al. [8]. Pooled standard deviation was calculated as per the Cochrane Handbook [9]. Standard deviation was calculated from mean and $p$ value using the RevMan calculator [10]. Odds ratio was converted to standardized mean difference as per Hasselblad and Hedges [11]. Calculation of statistics and meta-analysis were performed using StatsDirect [12] and Meta-Essentials [13]. Random effects models (DerSimonian Laird method) were utilized due to the heterogeneity of the interventions among studies.

Assessment of bias was performed using the Cochrane risk of bias assessment tool [9]. Two researchers (A.M. and S.N.) assessed risk of bias of each included study in 6 domains: random sequence generation; allocation concealment; blinding of participant; blinding of outcome assessment; incomplete outcome; and selective reporting. Any discrepancies were resolved by a third researcher (V.B.). Publication bias for the main outcomes was analysed by Egger's test [14] calculated by StatsDirect.

\section{Results}

The literature search process is summarized below in Figure 1. After screening of titles and abstracts, 19 publications were identified. Fifteen publications involving 12 cohorts were included in the final analysis, these are summarized in Table 1 [15-29]; 4 were excluded (3 due to no available data [30-32], 1 due to lack of randomization [33]). Seven publications included outcomes that could be analysed in meta-analysis.

\section{Baseline Population}

The total number of participants was 9,255, 8,003 (86\%) of whom were in 1 trial (Roffe et al. [15]). The mean age of participants (where provided) was 72.2 years. The proportion of patients with ischaemic stroke was $82.8 \%$ (range 61-100\%); the rate of stroke mimics was only declared in the Roffe et al. [15] study (3.5\%). Mean NIHSS across all patients was 7 . Oxygen regimes ranged from 2 $\mathrm{L} / \mathrm{min}$ via nasal cannula nocturnally to $45 \mathrm{~L} / \mathrm{min}$ via facemask for $8 \mathrm{~h}$ from randomization. Ninety-seven per cent of participants were involved in studies of low-flow oxygen $(\leq 4 \mathrm{~L} / \mathrm{min})$. Most protocols aimed to begin treatment 
Fig. 1. Literature search.

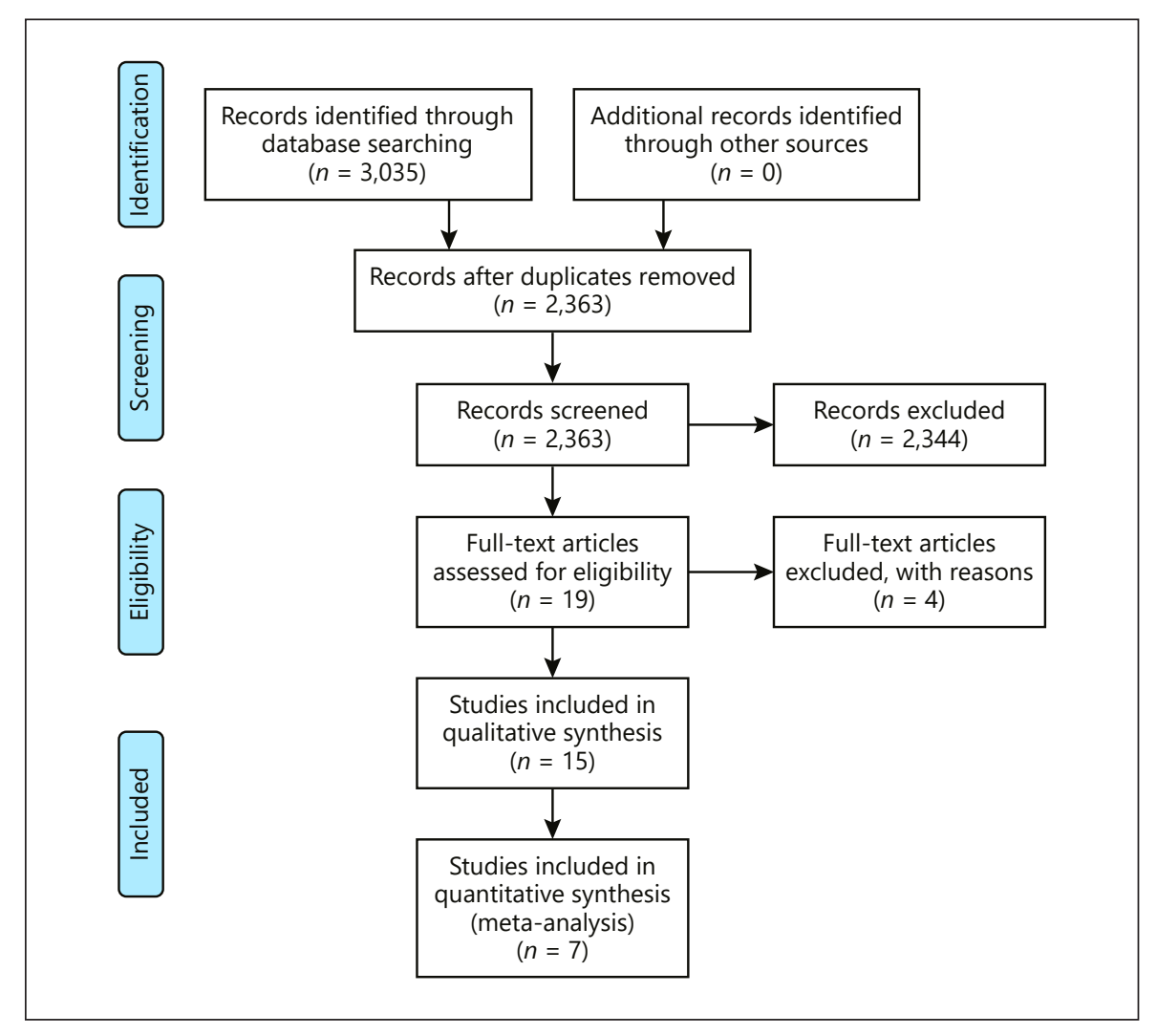

within the first $24 \mathrm{~h}$ following symptom onset. The median time to treatment was $19.3 \mathrm{~h}$. A summary of trial protocols is presented in Table 2.

\section{Bias Assessment}

The risk of bias assessment is shown in Table 3. Many studies had high risk of bias in at least one domain although the Roffe et al. [15] trial which enrolled the majority of patients did not. Insufficient methodological detail was published to allow determination of risk of bias for a number of smaller studies. Singhal [19] was not published and was evaluated on the basis of conference abstracts and a clinical trial registry. Egger's test had $p$ value $>0.05$ indicating a low risk of publication bias for all outcomes other than follow-up modified Rankin scale (mRS) though this result may be due to heterogeneity of studies rather than true publication bias. Funnel plot symmetry was seen in all other outcomes; however, the interpretation of this was cautious given the low number of studies.

\section{Outcomes}

Trials used similar and well-established measures of outcomes, but the timing of assessments varied. Trials broadly fell into 2 categories - large trials of low-flow oxygen used in the first $24-72 \mathrm{~h}$ primarily assessing functional outcomes; or smaller trials of high-flow oxygen used in the first $12-24 \mathrm{~h}$ primarily assessing early neurological recovery. Outcomes examined, the timing of their assessment, and numbers of participants for each are summarized in Table 4.

\section{Early Neurological Recovery}

Assessment of recovery in the first 7 days following stroke was measured using the National Institute of Health Stroke Scale (NIHSS). Six trials [15, 17, 18, 24, 27, 28], including the largest study, measured baseline and day 7 NIHSS. Meta-analysis (Fig. 2) of 5 of 6 studies demonstrated no significant difference in reduction in NIHSS at 7 days in all stroke (mean difference $=-0.16[-1.11$ to $0.80]$ ). The remaining study [27] was not included in the meta-analysis due to insufficient data. Two small trials $[19,28]$ measured NIHSS at early time points $(0,4$, and $24 \mathrm{~h}$ ); high-flow oxygen was found to improve NIHSS at $24 \mathrm{~h}$ in one study [28] but had no effect in the other [19]. Four studies also measured neurological recovery using the Scandinavian Stroke Scale (SSS), 2 studies $[20,23]$ in 


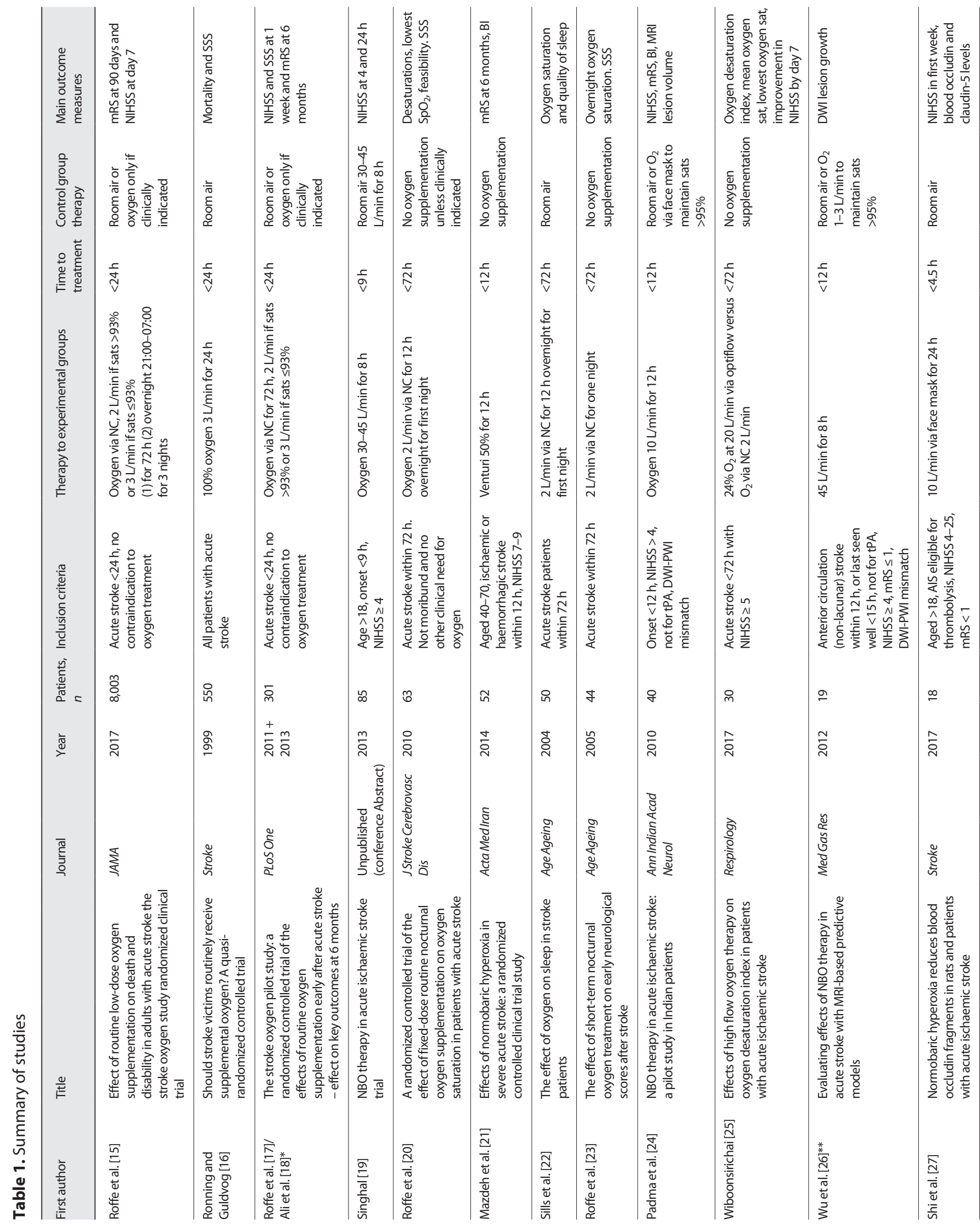




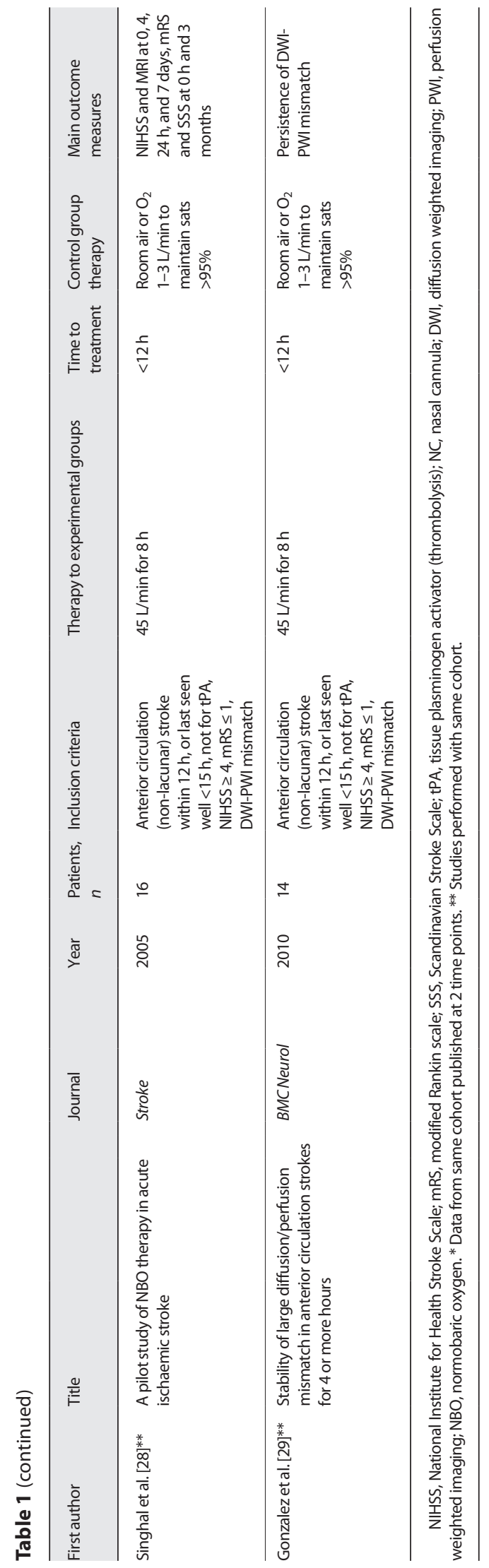

Normobaric Oxygen Therapy in Acute Stroke
Table 2. Summary of trial protocols

\begin{tabular}{lll}
\hline & Trials, $n$ & Participants, $n$ \\
\hline $\begin{array}{l}\text { Oxygen regimen } \\
\text { 2-3 L/min via nasal cannula }\end{array}$ & 6 & \\
$50 \%$ via Venturi mask & 1 & 9,011 \\
10-20 L/min via face mask & 3 & 52 \\
$30-45$ L/min via face mask & 4 & 104 \\
\hline Time to treatment & & \\
$<12, \mathrm{~h}$ & 7 & 214 \\
$<24, \mathrm{~h}$ & 3 & 8,854 \\
$<72, \mathrm{~h}$ & 4 & 187 \\
\hline
\end{tabular}

the early time period $(48 \mathrm{~h})$ and the others at 3 [28] and 7 months [16], respectively. No study found a difference in recovery measured by SSS between oxygen and control.

\section{Functional Outcome}

Longer term outcomes in functional ability following stroke were measured using mRS and Barthel Index (BI). $\mathrm{mRS}$ was measured at 3 months in 3 studies $[15,24,28]$ and 6 months in 2 studies $[18,21]$. Meta-analysis of 4 studies (the 5th [24] had insufficient data) for each time point and combined for both time points found no effect of NBO on mRS outcome (combined standardized mean difference $[\mathrm{SMD}]=-0.08[-0.38$ to 0.22$] ; 3$-month $\mathrm{SMD}=-0.01[-0.03$ to 0.029$] ; 6$-month $\mathrm{SMD}=-0.20$ [ -1.49 to 1.09$])$. BI was measured at 3 months $[15,24], 6$ months $[17,18,21]$, or 7 months [16] with no significant effect of NBO on BI outcome found in any study.

\section{Mortality}

Mortality was assessed at 7 days [15], 3 months [15], 6 months $[18,21]$, and 1 year [16]. Meta-analysis for combined 3-, 6-, and 12-month data demonstrated no significant effect of NBO on mortality (odds ratio $=1.15[0.87-$ 1.53]). One study [19] was stopped early due to excess mortality in the NBO group; however, a blinded reviewer concluded that 1 death in each group was "probably" related to treatment allocation and all other deaths were unrelated.

\section{Oxygen Saturation}

Baseline oxygen saturation was $>94 \%$ in 4 studies [ 15 , $18,20,23]$. Oxygen therapy was associated with raised oxygen saturation [15], reduced likelihood of desaturation [20], and higher nocturnal oxygen saturation [20, 22, 23]. 

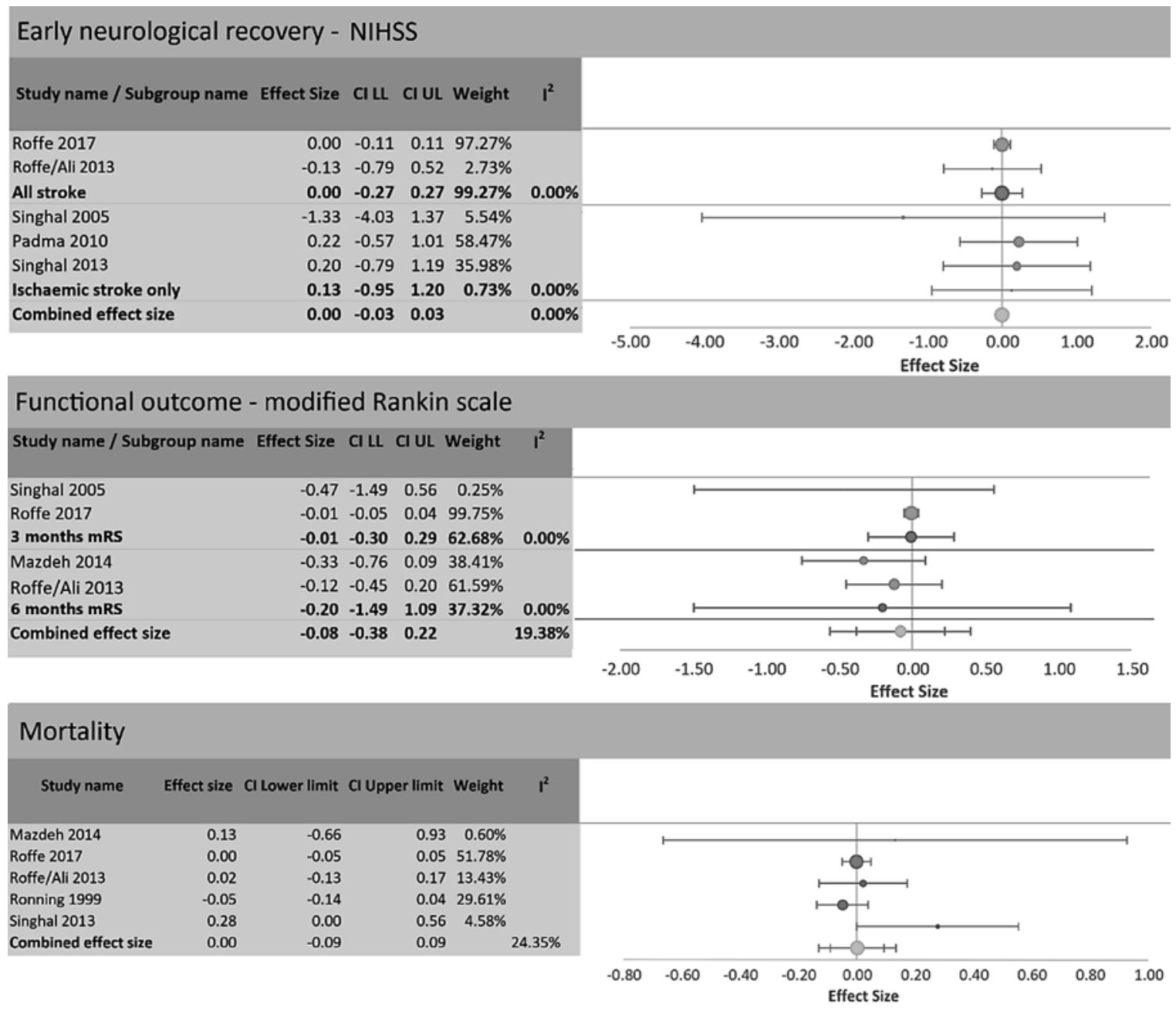

Fig. 2. Meta-analysis - left shift favours NBO, right shift favours control. NBO, normobaric oxygen; NIHSS, National Institute of Health Stroke Scale; mRS, modified Rankin scale; LL, lower limit; UL, upper limit.

Table 3. Risk of bias assessment

\begin{tabular}{|c|c|c|c|c|c|c|}
\hline & $\begin{array}{l}\text { Random sequence } \\
\text { generation }\end{array}$ & $\begin{array}{l}\text { Allocation } \\
\text { concealment }\end{array}$ & $\begin{array}{l}\text { Blinding of } \\
\text { participant }\end{array}$ & $\begin{array}{l}\text { Blinding of outcome } \\
\text { assessment }\end{array}$ & $\begin{array}{l}\text { Incomplete } \\
\text { outcome }\end{array}$ & $\begin{array}{l}\text { Selective } \\
\text { reporting }\end{array}$ \\
\hline Roffe et al. [15] & Low & Low & Low & Low & Low & Low \\
\hline Roffe et al. [17]/Ali et al. [18] & Low & Low & Unknown & Unknown & Low & Low \\
\hline Singhal [19] & Unknown & Low & Low & Low & Low & Low \\
\hline Roffe et al. [20] & Low & Low & Low & High & Low & Low \\
\hline Mazdeh et al. [21] & Unknown & Unknown & High & High & High & High \\
\hline Padma et al. [24] & Low & Unknown & Low & High & Unknown & High \\
\hline Wiboonsirichai [25] & Unknown & Unknown & Low & High & Unknown & Unknown \\
\hline Wu et al. [26] & Low & Low & Low & Low & Low & Low \\
\hline Shi et al. [27] & High & Unknown & High & High & Unknown & Unknown \\
\hline Singhal et al. [28] & Low & Low & Low & Low & Low & Low \\
\hline Gonzalez et al. [29] & Low & Low & Low & Low & Low & Low \\
\hline
\end{tabular}




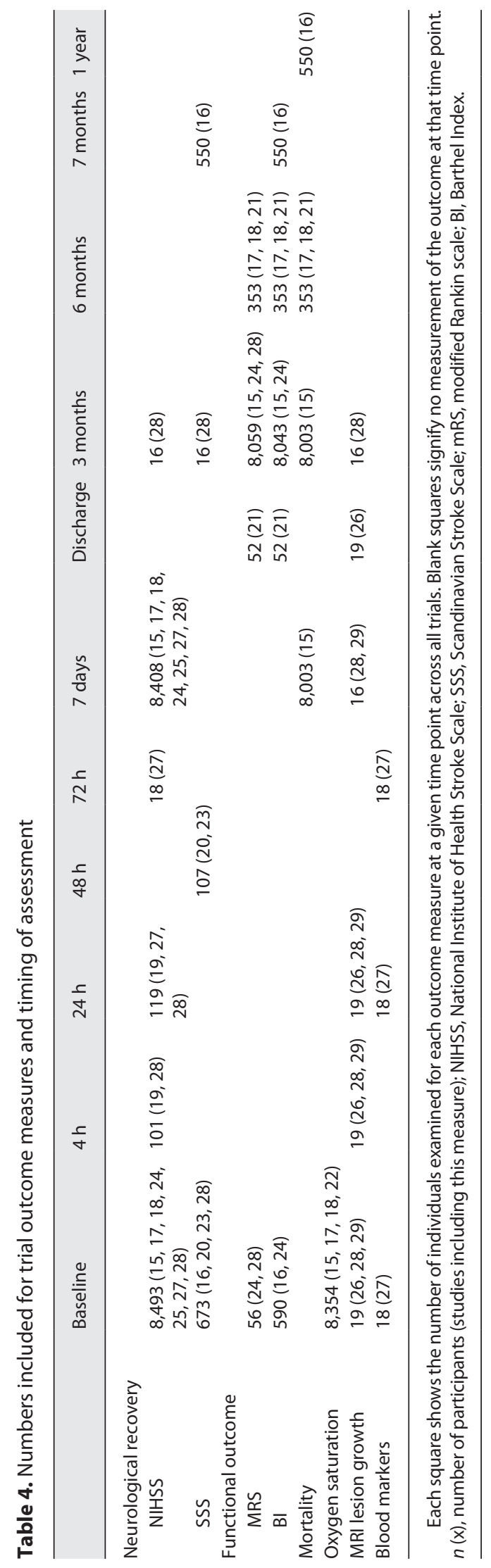

Normobaric Oxygen Therapy in Acute Stroke

\section{Imaging Outcomes}

Imaging outcomes were studied in 2 cohorts [19, 26, $28,29]$, reporting reduction in DWI lesion volume with $\mathrm{NBO}$ at $4 \mathrm{~h}$ but not $24 \mathrm{~h}$ in one study [28], and no significant difference in DWI lesion growth in the other cohort [19].

\section{Adverse Events}

Three trials reported adverse outcomes. In the largest trial of 8,003 patients [15], no oxygen-related adverse events were reported and the total number of serious adverse events was lower in the nocturnal oxygen group than in the continuous oxygen and control groups. In a study of 289 patients [17], there was no significant difference in peak heart rate, peak blood pressure, peak temperature, or proportions requiring antibiotics or sedatives. One study [19] was stopped early due to excess mortality in the experimental group; however, independent review determined that 1 death in each group was related to the intervention and all other deaths were unrelated.

\section{Subgroup Analysis}

The majority of studies reported outcomes jointly for ischaemic and haemorrhagic stroke though 3 studies examined ischaemic stroke only $[19,24,28]$, all of which also used high-flow oxygen. The only outcome reported with sufficient data across all 3 studies was NIHSS at baseline and 7 days. The difference in reduction in NIHSS at 7 days was not significant (mean difference $-0.73[-3.54$ to 2.08], respectively).

\section{Discussion}

Our systematic review and meta-analysis found no effect of NBO on any outcome, including early neurological improvement and functional outcome at 3-6 months. Protocols varied widely amongst trials, but the majority of data (6/12 trials and 9,011/9,255 patients) relate to low-flow oxygen $(2-4 \mathrm{~L} / \mathrm{min}$ via nasal cannula) commenced predominantly in the $12-24 \mathrm{~h}$ period after stroke onset, establishing the safety of low-flow oxygen in acute stroke patients. Animal data have explored the potential for NBO supplementation to improve tissue oxygenation in the ischaemic penumbra as a means of delaying irreversible metabolic failure in ischaemic tissue pending definitive reperfusion, a concept termed "penumbral freezing" [34]. Completed clinical trials have not adequately tested this concept, having delivered insufficient supplementary oxygen predominantly at time points well be- 
yond those when substantial volumes of penumbra typically persist.

Advantages of therapeutic oxygen supplementation over conventional pharmacological approaches to neuroprotection include low cost; familiarity and universal availability in healthcare systems; few adverse effects and contraindications; and potential for application in prehospital care. Use of oxygen carrying molecules such as perfluorocarbons (PFCs) could further increase oxygen delivery to ischaemic tissues. Previous animal studies of PFCs combined with NBO in models of transient and permanent middle cerebral artery occlusion have found reduction in infarct size $[35,36]$ or delay in infarct growth [37], though infarct size was determined very early (4-6 h) in some of the studies. Further animal studies have demonstrated combined use of PFCs and NBO to identify areas of penumbral tissue using T2* MRI blood oxygen level dependent imaging in a combined therapeutic and diagnostic approach [38]. Early phase human trials of an alternative PFC (administered without NBO) have demonstrated safety and further trials are planned [39].

Reperfusion therapies address the underlying cause of tissue ischaemia but the requirement for diagnostic confirmation and imaging, currently overwhelmingly hospital-based, incurs inevitable delay. The time from symptom onset to initiation of treatment was $240 \pm 72 \mathrm{~min}$ in a meta-analysis of the alteplase thrombolysis trials, [40], and the time period from symptom onset to arterial puncture was $250.4 \pm 95.6 \mathrm{~min}$ in the mechanical thrombectomy trials of the HERMES group [41]. Dependent on factors including clot burden, reperfusion may not be achieved for some time after treatment initiation, and both drug treatment and thrombectomy may fail to effect reperfusion in a moderate to high proportion of patients treated. In countries with well-equipped ambulance services, delivery of NBO is feasible from the point of provisional diagnosis in the field and could be continued during hospital transfers whilst suitability for reperfusion therapies is determined by clinical evaluation and neuroimaging. Pre-hospital paramedic-delivered clinical trials in acute stroke have been shown to be feasible in the UK (RIGHT-2 [42]) and USA (FAST-MAG [43]) though rates of mimics can be high ( $26 \%$ in RIGHT-2 compared with $3.9 \%$ in FAST-MAG) and a significant proportion have haemorrhagic stroke (13\% in RIGHT-2, $22.8 \%$ in FAST-MAG). The safety of NBO in haemorrhagic stroke and common mimics would ideally need to be established in this pre-hospital setting, but there is wide experience of supplemental oxygen in a broad range of clinical situations; animal studies of NBO in haemorrhagic stroke have shown no effect [44] or clinical improvement [45] compared to control. Oxygen saturations have been found to decrease during intra-hospital transfers, e.g., to CT or MRI scanning [46]. Inter-hospital transfers are commonly required in order to access mechanical thrombectomy, often following initiation of thrombolysis. Animal studies have suggested NBO and thrombolysis are safe in combination [6]. Any potential "penumbral freezing" effect of NBO would be advantageous for patients travelling to another site for mechanical thrombectomy.

Of the participants included in the trials in this review only $2 \%(n=192)$ received oxygen over $10 \mathrm{~L} / \mathrm{min}$. The largest trial of high-flow oxygen [19] ( $>30 \mathrm{~L} / \mathrm{min})$ in a hyperacute population was halted early due to excess mortality in the experimental group; however, independent review determined that the effect was not due to the intervention. Some concerns have been raised previously about the potential toxicity of high-flow oxygen in the acute stroke period due to factors such as formation of reactive oxygen species [47] and risk of hyperoxic acute lung injury [48]. While hyperoxia causes cerebral vasoconstriction, this does not reduce brain perfusion significantly in healthy individuals [49]. Whether hyperoxic vasoconstriction may compromise critically perfused brain regions in the setting of acute ischaemic stroke or intracerebral haemorrhage is uncertain, and the balance of any change in blood flow against increased oxygen delivery is challenging to determine. A study [50] of patients intubated prior to mechanical thrombectomy for acute ischaemic stroke with subsequent intensive care admission found patients with $\mathrm{PaO}_{2}>120 \mathrm{~mm} \mathrm{Hg}(16 \mathrm{kPa})$ had a poorer functional outcome as measured by the mRS than those $<120 \mathrm{~mm} \mathrm{Hg}$. This was, however, an observational study. A recent systematic review [47] identified that unfavourable outcome had been found in retrospective studies of intracerebral haemorrhage or subarachnoid haemorrhage but not ischaemic stroke and there were few prospective studies. The tolerability of high-flow oxygen via mask compared to low-flow oxygen via nasal cannula is poorer $[51,52]$ and may present an additional challenge.

Only $2.3 \%$ of participants were in trials aiming to deliver NBO within $12 \mathrm{~h}$ of symptom onset. Median interval from stroke onset to treatment initiation was $19 \mathrm{~h}$. As highlighted above the period from symptom onset to initiation of revascularisation therapy has been approximately $4 \mathrm{~h}$ in clinical trials meaning that based on current data on NBO we are unable to draw conclusions regarding its potential role in penumbral freezing and neuroprotection. Initiation of treatment beyond the hyperacute 
window has been proposed as the primary reason for the neutral results of trials of neuroprotective agents in stroke to date [53].

Additionally, the inclusion criteria for the majority of the trials permitted a wide selection of acute stroke patients including haemorrhagic stroke. In order to test the penumbral freezing hypothesis, selection of reperfusioneligible patients with appropriate imaging features likely offers the most efficient design for future trials such as proposed in the upcoming PROOF trial [54] which will select patients with large vessel occlusion and compare $\mathrm{NBO} \geq 40 \mathrm{~L} / \mathrm{min}$ versus standard care within $3 \mathrm{~h}$ of symptom onset. Completed trials lack sufficient data on physiological brain imaging and timing or effectiveness of reperfusion therapies (if permitted) to allow even exploratory analysis.

The major limitation of this systematic review and meta-analysis is the large proportion of participants contributed by a single trial. Meta-analysis therefore largely reflects the results of this trial. Analyses of other sub-groups such as hyperacute populations or high-flow oxygen are limited by the small numbers in trials using such protocols. Detailed methodology was not available for several smaller clinical trials some of which had a high risk of bias often due to lack of blinding in outcome assessment. These factors highlight the lack of data for high-flow oxygen during the hyperacute period after stroke onset.

\section{Conclusions}

Studies to date of NBO have shown no significant effect on early recovery, functional outcome, or mortality in acute stroke. Study protocols and populations have varied considerably. Populations have included signifi- cant numbers of non-ischaemic stroke and stroke mimics. Oxygen has been predominantly low flow commenced in the sub-acute phase. Further study of highflow oxygen in the hyperacute period may be warranted.

\section{Statement of Ethics}

The article is exempt from Ethical Committee approval as it is a systematic review of publicly available data.

\section{Conflict of Interest Statement}

The authors have no conflicts of interest to declare.

\section{Funding Sources}

A.M. has received funding from Wellcome Trust.

\section{Author Contributions}

A.M. and S.N. carried out the literature search and identified suitable studies. V.B. resolved any conflicts in study inclusion. A.M. developed the protocol, carried out the analysis, and wrote the first draft. K.M. reviewed and edited the protocol and drafts. All authors reviewed and approved the final version of the manuscript.

\section{Data Availability Statement}

The data that support the findings of this study are openly availablein Open ScienceFrameworkathttps://osf.io/q4sv2/?view_ only=6f96ca6aaba24d9f9bef0b76c187ac58.

\section{References}

1 Kobayashi A, Czlonkowska A, Ford GA, Fonseca AC, Luijckx GJ, Korv J, et al. European Academy of Neurology and European Stroke Organization consensus statement and practical guidance for pre-hospital management of stroke. Eur J Neurol. 2018; 25(3):425-33.

2 Powers WJ, Rabinstein AA, Ackerson T, Adeoye OM, Bambakidis NC, Becker K, et al. 2018 Guidelines for the early management of patients with acute ischemic stroke: a guideline for healthcare professionals from the American Heart Association/American Stroke Association. Stroke. 2018;49(3):e4699.

Normobaric Oxygen Therapy in Acute Stroke
3 Ejaz S, Emmrich JV, Sitnikov SL, Hong YT, Sawiak SJ, Fryer TD, et al. Normobaric hyperoxia markedly reduces brain damage and sensorimotor deficits following brief focal ischaemia. Brain. 2016;139(Pt 3):751-64.

4 Kim HY, Singhal AB, Lo EH. Normobaric hyperoxia extends the reperfusion window in focal cerebral ischemia. Ann Neurol. 2005; 57(4):571-5.

5 Hadjiev DI, Mineva PP. Transient ischemic attack may present a target for normobaric hyperoxia treatment. Med Hypotheses. 2010; 75(1):128-30

6 Fujiwara N, Murata Y, Arai K, Egi Y, Lu J, Wu $\mathrm{O}$, et al. Combination therapy with normo- baric oxygen (NBO) plus thrombolysis in experimental ischemic stroke. BMC Neurosci. 2009; 10:79.

7 Hozo SP, Djulbegovic B, Hozo I. Estimating the mean and variance from the median, range, and the size of a sample. BMC Med Res Methodol. 2005;5:13.

8 Luo D, Wan X, Liu J, Tong T. Optimally estimating the sample mean from the sample size, median, mid-range, and/or mid-quartile range. Stat Methods Med Res. 2018;27(6): 1785-805.

9 Higgins JPT, Green S. Cochrane handbook for systematic reviews of interventions. London: The Cochrane Collaboration; 2011. 
10 The Cochrane Collaboration. Review manager (RevMan) [computer program]. Version 5.3. 2014.

11 Borenstein M, Hedges LV, Higgins JPT, Rothstein HR. Introduction to meta-analysis. Hoboken: John Wiley \& Sons; 2009.

12 StatsDirect. StatsDirect statistical software. StatsDirect Ltd; 2013. Available from: http: //www.statsdirect.com.

13 Suurmond R, van Rhee H, Hak T. Introduction, comparison, and validation of metaessentials: a free and simple tool for metaanalysis. Res Synth Methods. 2017;8(4): 537-53.

14 Egger M, Davey Smith G, Schneider M, Minder C. Bias in meta-analysis detected by a simple, graphical test. BMJ. 1997; 315(7109):629-34.

15 Roffe C, Nevatte T, Sim J, Bishop J, Ives N, Ferdinand P, et al. Effect of routine lowdose oxygen supplementation on death and disability in adults with acute stroke: the stroke oxygen study randomized clinical trial. JAMA. 2017;318(12):1125-35. Erratum in: JAMA. 2017 Nov 14;318(18):183132.

16 Ronning OM, Guldvog B. Should stroke victims routinely receive supplemental oxygen? A quasi-randomized controlled trial. Stroke. 1999;30(10):2033-7.

17 Roffe C, Ali K, Warusevitane A, Sills S, Pountain S, Allen M, et al. The SOS pilot study: a RCT of routine oxygen supplementation early after acute stroke: effect on recovery of neurological function at one week. PLoS One. 2011;6(5):e19113.

18 Ali K, Warusevitane A, Lally F, Sim J, Sills $S$, Pountain S, et al. The stroke oxygen pilot study: a randomized controlled trial of the effects of routine oxygen supplementation early after acute stroke-effect on key outcomes at six months. PLoS One. 2013;8(6): e59274.

19 Singhal A. A phase iib clinical trial of normobaric oxygen therapy (NBO) in acute ischemic stroke (AIS). Neurology. Conference: 65th American Academy of Neurology Annual Meeting. San Diego, CA. Conference Publication: 2013. 2013;80(1 MeetingAbstracts).

20 Roffe C, Sills S, Pountain SJ, Allen M. A randomized controlled trial of the effect of fixed-dose routine nocturnal oxygen supplementation on oxygen saturation in patients with acute stroke. J Stroke Cerebrovasc Dis. 2010;19(1):29-35.

21 Mazdeh M, Taher A, Torabian S, Seifirad S. Effects of normobaric hyperoxia in severe acute stroke: a randomized controlled clinical trial study. Acta Med Iran. 2015;53(11): 676-80.

22 Sills S, Roffe C, Halim M. The effect of oxygen on sleep in stroke patients. Age Ageing. 2004;33(Suppl 2):ii42.
23 Roffe C, Sills S, Halim M. The effect of short-term nocturnal oxygen treatment on early neurological scores after stroke. Age Ageing. 2005;34(Suppl 1):i45.

24 Padma MV, Bhasin A, Bhatia R, Garg A, Singh MB, Tripathi $M$, et al. Normobaric oxygen therapy in acute ischemic stroke: a pilot study in Indian patients. Ann Indian Acad Neurol. 2010;13(4):284-8.

25 Wiboonsirichai N, Kongpolprom N, Desudchit T, Sittipunt C. Effects of high flow oxygen therapy on oxygen desaturation index in patients with acute ischemic stroke. Respirology. Conference: 22nd Congress of the Asian Pacific Society of Respirology. ASPR. 2017. Australia. 2017;22(Suppl 3): 38.

$26 \mathrm{Wu}$ O, Villien M, Thankachan T, Mocking S, Copen WA, Schaefer PW, et al. Functional MRI and CBF responses to transient oxygen challenges in acute ischemic stroke patients. Stroke. Conference. 2014;45(Suppl 1):AWP33.

27 Shi S, Qi Z, Ma Q, Pan R, Timmins GS, Zhao $\mathrm{Y}$, et al. Normobaric hyperoxia reduces blood occludin fragments in rats and patients with acute ischemic stroke. Stroke. 2017;48(10):2848-54.

28 Singhal AB, Benner T, Roccatagliata L, Koroshetz WJ, Schaefer PW, Lo EH, et al. A pilot study of normobaric oxygen therapy in acute ischemic stroke. Stroke. 2005; 36(4):797-802.

29 Gonzalez RG, Hakimelahi R, Schaefer PW, Roccatagliata L, Sorensen AG, Singhal AB. Stability of large diffusion/perfusion mismatch in anterior circulation strokes for 4 or more hours. BMC Neurol. 2010;10:13.

30 Cheng Z. A study on treatment of acute ischemic stroke with endovascular therapy and normobaric oxygenation. 2017.

31 Mint P. Randomised controlled study of the effect of low flow oxygen on capillary blood gases after acute stroke. 2018 [cited 2021 Oct 25]. Available from: https://www.isrctn.com/ISRCTN75718175.

32 Xunming J. Protective effects of normobaric hyperoxia on blood brain barrier in patients with acute ischemic stroke. 2016 [cited 2021 Oct 25]. Available from: https:// clinicaltrials.gov/ct2/show/NCT02974283.

33 Chiu EH, Liu C-S, Tan T-Y, Chang K-C. Venturi mask adjuvant oxygen therapy in severe acute ischemic stroke. Arch Neurol. 2006;63(5):741-4.

34 Baron JC. Protecting the ischaemic penumbra as an adjunct to thrombectomy for acute stroke. Nat Rev Neurol. 2018;14(6): 325-37.

35 Seiffge DJ, Lapina NE, Tsagogiorgas C, Theisinger B, Henning RH, Schilling L. Improvement of oxygen supply by an artificial carrier in combination with normobaric oxygenation decreases the volume of tissue hypoxia and tissue damage from transient focal cerebral ischemia. Exp Neurol. 2012; 237(1):18-25.
36 Brown AT, Arthur MC, Nix JS, Montgomery JA, Skinner RD, Roberson PK, et al. Dodecafluoropentane emulsion (DDFPe) decreases stroke size and improves neurological scores in a permanent occlusion rat stroke model. Open Neurol J. 2014;8:27-33.

37 Fitzgerald RT, Ou X, Nix JS, Arthur MC, Brown AT, Skinner RD, et al. Dodecafluoropentane emulsion delays and reduces MRI markers of infarction in a rat stroke model: a preliminary report. Magn Reson Imaging. 2015;33(2):236-9.

38 Deuchar GA, Brennan D, Holmes WM, Shaw M, Mhairi Macrae I, Santosh C. Perfluorocarbon enhanced glasgow oxygen level dependent (GOLD) magnetic resonance metabolic imaging identifies the penumbra following acute ischemic stroke. Theranostics. 2018;8(6):1706-22.

39 Brown A, McGonigle J, Graham K, Unger E, et al. Abstract WP120: welcoming the new kid on the block; the phase IIb prospective, randomized, open-label, blinded endpoint (PROBE) study of NanO2TM neuroprotection in large vessel strokes. Stroke. 2020;51(Suppl 1):AWP120.

40 Emberson J, Lees KR, Lyden P, Blackwell L, Albers G, Bluhmki E, et al. Effect of treatment delay, age, and stroke severity on the effects of intravenous thrombolysis with alteplase for acute ischaemic stroke: a metaanalysis of individual patient data from randomised trials. Lancet. 2014;384(9958): 1929-35.

41 Bourcier R, Goyal M, Liebeskind DS, Muir KW, Desal H, Siddiqui AH, et al. Association of time from stroke onset to groin puncture with quality of reperfusion after mechanical thrombectomy: a meta-analysis of individual patient data from 7 randomized clinical trials. JAMA Neurol. 2019; 76(4):405-11.

42 Bath PM, Scutt P, Anderson CS, Appleton JP, Berge E, Cala L, et al. Prehospital transdermal glyceryl trinitrate in patients with ultra-acute presumed stroke (RIGHT-2): an ambulance-based, randomised, sham-controlled, blinded, phase 3 trial. Lancet. 2019; 393(10175): 1009-20.

43 Saver JL, Starkman S, Eckstein M, Stratton SJ, Pratt FD, Hamilton S, et al. Prehospital use of magnesium sulfate as neuroprotection in acute stroke. N Engl J Med. 2015; 372(6):528-36.

44 Fujiwara N, Mandeville ET, Geng X, Luo Y, Arai K, Wang X, et al. Effect of normobaric oxygen therapy in a rat model of intracerebral hemorrhage. Stroke. 2011;42(5):146972.

45 You P, Lin M, Li K, Ye X, Zheng J. Normobaric oxygen therapy inhibits HIF-1 $\alpha$ and VEGF expression in perihematoma and reduces neurological function defects. Neuroreport. 2016;27(5):329-36. 
46 Rowat AM, Dennis MS, Wardlaw JM. Hypoxaemia in acute stroke is frequent and worsens outcome. Cerebrovasc Dis. 2019; 21(3):166-72.

47 Stolmeijer R, Bouma HR, Zijlstra JG, Drostde Klerck AM, Ter Maaten JC, Ligtenberg JJM. A systematic review of the effects of hyperoxia in acutely Ill patients: should we aim for less? Biomed Res Int. 2018;2018: 7841295.

48 Kallet RH, Matthay MA. Hyperoxic acute lung injury. Respir Care. 2013;58(1):12341.
49 Bulte DP, Chiarelli PA, Wise RG, Jezzard P. Cerebral perfusion response to hyperoxia. J Cereb Blood Flow Metab. 2007;27(1):6975.

50 Lopez HV, Vivas MF, Ruiz RN, Martínez JR, Navaridas BG-V, Villa MG, et al. Association between post-procedural hyperoxia and poor functional outcome after mechanical thrombectomy for ischemic stroke: an observational study. Ann Intensive Care. 2019;9(1):59.

51 Costello RW, Liston R, McNicholas WT. Compliance at night with low flow oxygen therapy: a comparison of nasal cannulae and Venturi face masks. Thorax. 1995; 50(4):405-6.
52 Ayhan H, Iyigun E, Tastan S, Orhan ME, Ozturk E. Comparison of two different oxygen delivery methods in the early postoperative period: randomized trial. J Adv Nurs. 2009;65(6):1237-47.

53 Saver JL. The 2012 Feinberg Lecture: treatment swift and treatment sure. Stroke. 2013;44;270-7.

54 Poli S. Penumbral rescue by normobaric $\mathrm{O} 2$ administration in patients with ischemic stroke and target mismatch profile - full text view - ClinicalTrials.gov. 2019. Available from: https://clinicaltrials.gov/ct2/ show/NCT03500939. 